\title{
PRODUÇÃO CIENTÍFICA BRASILEIRA EM COMPORTAMENTO ORGANIZACIONAL NO PERIODO 2000-2010
}

\author{
BRAZILIAN SCIENTIFIC PRODUCTION IN ORGANIZATIONAL BEHAVIOR IN THE PERIOD \\ 2000-2010 \\ PRODUCCIÓN CIENTÍFICA BRASILEÑA SOBRE COMPORTAMIENTO ORGANIZACIONAL EN EL PERÍODO 2000- \\ 2010
}

\section{RESUMO}

O presente artigo tem como objetivo fazer uma análise da produção científica em Comportamento Organizacional (CO), com base nos principais periódicos brasileiros de Administração e anais do Encontro Nacional da Associação Nacional de Pós-graduação e Pesquisa em Administração (EnANPAD). No total, foram analisados 185 artigos publicados em periódicos e 696 no EnANPAD, de 2001 a 2010. As análises foram baseadas no levantamento das temáticas e procedimentos metodológicos utilizados. Os resultados encontrados contribuem para uma melhor delimitação do campo de CO e caracterização do seu escopo temático. Os resultados apontam para uma identidade própria da produção acadêmica brasileira de $\mathrm{CO}$, com destaque para temas com enfoque macro, como cultura e aprendizagem organizacional, apesar de o campo evidenciar uma grande diversidade de temas. Quanto aos aspectos metodológicos, verifica-se que predomina uma abordagem qualitativa e destacam-se algumas fragilidades metodológicas. Por outro lado, é importante realçar que o campo tem crescido nos últimos anos e que se verifica um aumento na variedade de estratégias de pesquisa e na utilização de técnicas de análise de dados mais sofisticadas.

PALAVRAS-CHAVE Comportamento organizacional, produção intelectual, estratégias de pesquisa, métodos de coleta de dados, métodos de tratamento de dados.

Filipe João Bera de Azevedo Sobral filipe.sobral@fgv.br

Professor da Escola Brasileira de Administração Pública e de Empresas, Fundação Getulio Vargas - Rio de Janeiro - RJ, Brasil

Juliana Arcoverde Mansur juliana.mansur@fgvmail.br

Doutoranda em Administração de Empresas pela Escola Brasileira de Administração Pública e de Empresas, Fundação Getulio Vargas - Rio de Janeiro - RJ, Brasil

\begin{abstract}
The aim of this article is to conduct an analysis of scientific production in Organizational Behavior (OB) based on the leading Brazilian periodicals and annals of (EnANPAD). Altogether, 185 published articles in periodicals were analyzed and 696 in EnANPAD from 2001 to 2010. The analyses were based on surveys of the methodological approaches and procedures employed. The results obtained made it possible to set out the boundaries of the field of $\mathrm{CO}$ and characterize its range of objectives. They also established that the academic production of $\mathrm{CO}$ in Brazil has its own identity and highlights issues of a macro kind, such as culture and organizational learning, although there is evidence that the field encompasses a wide variety of areas. With regard to methodological factors, a qualitative approach is found to be predominant and some stress is laid on methodological weaknesses. At the same time, it should be underlined that the field has grown in recent years and has witnessed an increase in the range of research strategies and the employment of more sophisticated techniques for data analysis. keywords Organizational behavior, intellectual production, methodology, methods of data collection, data processing methods.

Resumen El presente artículo tiene como objetivo hacer un análisis de la producción científica sobre Comportamiento Organizacional (CO), con base en los principales periódicos brasileños de administración y anales del (EnANPAD). En total, fueron analizados 185 artículos publicados en periódicos y 696 en el EnANPAD, de 2001 a 2010. Los análisis se basaron en el relevamiento de las temáticas y procedimientos metodológicos utilizados. Los resultados encontrados contribuyen a una mejor delimitación del campo del CO y caracterización de su esfera temática. Los resultados apuntan hacia una identidad propia de la producción académica brasileña sobre el CO, principalmente hacia temas con enfoque macro, como cultura y aprendizaje organizacional, a pesar de que el campo evidencie una gran diversidad de temas. Respecto a los aspectos metodológicos, se verifica que predomina un abordaje cualitativo y se destacan algunas fragilidades metodológicas. Por otro lado, es importante realzar que el campo ha crecido en los últimos años y que se verifica un aumento en la variedad de estrategias de investigación y en la utilización de técnicas de análisis de datos más sofisticadas.
\end{abstract}

Palabras clave Comportamiento organizacional, producción intelectual, metodología, métodos de recogida de datos, métodos de procesamiento de datos 


\section{INTRODUÇÃO}

Desde que o Comportamento Organizacional (CO) foi reconhecido como campo de estudos, em meados dos anos 1960, muitas definições foram propostas para explicar e determinar seus limites e níveis estruturais. $\mathrm{CO}$ pode ser entendido tanto como um campo que examina o comportamento de ambientes organizacionais quanto uma área de investigação sobre a influência que indivíduos, grupos e estrutura organizacional exercem sobre o comportamento nas organizações. Ao longo desses 50 anos de desenvolvimento, o CO tem-se consolidado como uma área de teorização e pesquisa central para o conhecimento administrativo e gerencial, buscando analisar e compreender questões bastante complexas e abrangentes no âmbito das organizações.

O objetivo deste trabalho consiste em fazer um retrato dos últimos 10 anos da evolução do campo de CO no Brasil, baseado no levantamento sistemático, análise e avaliação crítica da produção científica no período de 2001 a 2010, tendo por base uma pesquisa documental nos principais periódicos de Administração ( $R A E, R A P, R A C, R A M$ e RAUSP) e nos anais do EnANPAD. O interesse principal do trabalho reside na compreensão de como essa área é vista e estudada por acadêmicos e pesquisadores da área de Administração.

\section{COMPORTAMENTO ORGANIZACIONAL: DISTINÇÕES E DELIMITAÇ̃̃ES}

O CO, definido como o campo de conhecimento interessado no estudo sistemático do comportamento dos indivíduos e dos grupos nas organizações, bem como da estrutura e do comportamento destas, resulta da confluência de saberes de diferentes áreas disciplinares que compartilham um interesse em comum: o estudo das organizações.

Entre as disciplinas que influenciaram a consolidação do campo de CO, destacam-se a psicologia, a sociologia, a antropologia, a ciência política e a economia. Entretanto, integrar disciplinas tão complexas e abrangentes, que muitas vezes não compartilham definições conceituais e têm divergências quanto aos paradigmas dominantes, é um enorme desafio do ponto de vista epistemológico e metodológico (MOWDAY e SUTTON, 1993; ROUSSEAU, 1997).
De maneira geral, as disciplinas que apoiam o campo de CO possuem objetos de estudo que trabalham o comportamento de indivíduos, grupos e organizações. No entanto, faz-se necessária uma clara distinção entre o campo de CO e dois campos específicos de conhecimento com os quais este compartilha tênues fronteiras: a Teoria Organizacional (TO) e a Gestão de Recursos Humanos (GRH).

A TO constitui uma disciplina próxima ao $\mathrm{CO}$ que tem por domínio específico a construção e teste de teorias sobre as organizações e sua gestão, e as relações entre a organização e o ambiente, privilegiando o nível macro de análise (STAW, 1984). Por sua vez, o CO privilegia um olhar micro sobre os fenômenos organizacionais, tendo o indivíduo como a sua menor unidade de análise, e preocupa-se com a compreensão do comportamento humano dentro das organizações (NORTHCRAFT e NEALE, 1990). Já o campo de GRH tem como interesse investigar as práticas e técnicas relativas à gestão de pessoas nos contextos organizacionais, caracterizando-se como um campo mais aplicado, focado nas ferramentas e técnicas de gestão de pessoas (BORGES-ANDRADE e PAGOTTO, 2010).

Apesar de conceitualmente distintos, os três campos ainda apresentam uma interseção significativa, compartilhando diversos interesses e temas de análise. Entretanto, este trabalho focará apenas o campo de $\mathrm{CO}$, excluindo trabalhos que, de acordo com as delimitações propostas, sejam classificados como de GRH e TO.

\section{CONSOLIDAÇÃO DO CAMPO DE COMPORTAMENTO ORGANIZACIONAL}

Desde o seu reconhecimento como campo de conhecimento, CO recebeu diversas conceituações. A ideia de constituir um campo independente de estudos, porém multidisciplinar, baseou-se no argumento de que as atividades na organização não poderiam ser consideradas como o contexto para o qual os fenômenos psicológicos simplesmente seriam transferidos e aplicados, como era defendido pela Psicologia Organizacional; pelo contrário, deveriam ser consideradas como o próprio objeto de estudo de um campo de conhecimento, que, então, seria apoiado pelas demais ciências do comportamento, como a Psicologia, a Sociologia e a Antropologia (SIQUEIRA, 2002). 
As primeiras tentativas para delimitar o campo de CO surgiram ainda no final da década de 1960, quando Pugh $(1966,1969)$ definiu CO como o estudo da estrutura e do funcionamento de organizações e do comportamento de grupos e indivíduos dentro delas. Posteriormente, Payne e Pugh (1971) apresentam um dos primeiros esquemas conceituais para o $\mathrm{CO}$, com quatro níveis de análise: indivíduos, equipes ou grupos de trabalho, departamentos ou outros pequenos setores organizacionais e a organização como um todo.

No entanto, foi a partir da década de 1970 que essa nova disciplina começou a ganhar espaço na academia, especialmente a partir de 1979, quando a Annual Review of Psychology começou a publicar periodicamente revisões sobre a evolução do campo de CO (exs: MITCHELL, 1979; MOWDAY e SUTTON, 1993; O'REILLY, 1991; ROUSSEAU, 1997; STAW, 1984), que garantiram a consolidação e independência do campo enquanto área de conhecimento independente.

Em 1984, Staw definiu o CO como o campo multidisciplinar que examina o comportamento individual dentro das organizações, além da estrutura e do comportamento das próprias organizações (STAW, 1984). Com a evolução e avanço das pesquisas no campo de CO, o escopo foi, então, estruturado em três níveis de análise a partir da década de 1990 - os indivíduos (micro), as equipes e grupos de trabalho (meso) e a organização como um todo (macro). O primeiro nível de análise, comportamento micro-organizacional, tem contribuições teóricas acentuadas da Psicologia e focaliza os aspectos psicossociais do indivíduo e as dimensões de sua atuação no contexto organizacional. O segundo, comportamento meso-organizacional, volta-se para questões relativas aos processos de grupos e equipes de trabalho, cuja compreensão teórica é oferecida por postulados da Antropologia, Sociologia e Psicologia Social. O terceiro nível de análise, comportamento macro-organizacional, com marcantes contribuições da Antropologia, Ciência Política e Sociologia, dá ênfase ao entendimento da organização como um todo, focando em temas como a estrutura, a cultura, a mudança e a aprendizagem organizacional.

No Brasil, o primeiro levantamento sobre produção acadêmica brasileira em Administração foi realizado na década de 1990, quando Machado-da-Silva, Cunha e Amboni (1990) realizaram a avaliação dos artigos da área de Organizações, analisando as publicações de 1985 a 1989 em periódicos. Com base na identificação de uma fragilidade teórico-metodológica e do predomínio funcionalista no período, outras pes- quisadores investiram na produção de balanços críticos desse tipo (ex: TONELLI e outros, 2003). Todos, no entanto, indicam problemas sérios de qualidade (BERTERO E KEINERT, 1994; BERTERO, CALDAS, WOOD, 1999; DAVEL e ALCADIPANI, 2003). Os autores questionavam, por exemplo, a aplicabilidade de conceitos, a metodologia utilizada e a importação de literatura.

Especificamente em relação à área de $\mathrm{CO}$, destacam-se cinco estudos dedicados ao exame da produção científica no Brasil (BASTOS e outros, 1997; BORGES-ANDRADE e outros, 1997; TONELLI e outros, 2003; TONETTO e outros, 2008; BORGES-ANDRADE e PAGOTTO, 2010). Os estudos revelam a predominância de temas como cultura organizacional, aprendizagem organizacional e comprometimento, evidenciando um equilíbrio entre as abordagens qualitativa e quantitativa.

Apesar de os estudos realizados, de certa forma, retratarem o campo de CO, têm as suas limitações, uma vez que nenhum analisa a produção do campo considerando os três níveis de análise: indivíduo (micro), grupos (meso) e organização per se (macro). Assim, o presente trabalho pretende colaborar para uma avaliação abrangente do campo de CO com base na análise de artigos publicados nos principais periódicos científicos de Administração e anais do EnANPAD, de 2001 a 2010.

\section{METODOLOGIA}

Considerando que a unidade de análise deste trabalho são publicações, os dados do presente estudo constituíram-se de artigos publicados entre 2001 e 2010 nos principais periódicos de Administração brasileiros: RAE-Revista de Administração de Empresas (RAE), Revista de Administração Contemporânea (RAC), Revista de Administração Pública (RAP), Revista de Administração da USP (RAUSP) e Revista de Administração Mackenzie (RAM), bem como no principal evento científico de Administração no Brasil, o EnANPAD. As publicações deveriam ter como objeto de estudo uma variável de CO. A inclusão ou exclusão das publicações na área de CO foi definida pela análise dos títulos, resumos e palavras-chave dos artigos publicados nos referidos periódicos e nas divisões de Estudos Organizacionais e Gestão de Pessoas e Relações do Trabalho nos anais da EnANPAD. Nos casos em que a leitura desses elementos não foi suficiente para definir se a publicação se enquadrava ou não 
nos critérios, realizou-se a leitura na íntegra do texto. Esse processo resultou num total de 185 artigos em periódicos e 696 no EnANPAD que se enquadravam no campo de CO, durante os 10 anos analisados. Foram excluídos da análise 27 trabalhos publicados nos anais do EnANPAD que foram posteriormente publicados em periódicos.

Depois de identificados todos os artigos que cumpriam os critérios de inclusão na análise, dois codificadores previamente treinados classificaram cada artigo conforme as categorias conceituais (ver Quadro 1), formuladas com base nas revisões sobre CO publicadas na literatura internacional, especificamente no Annual Review of Psychology e no Academy of Management Journal. Cada artigo foi classificado em uma ou duas categorias de análise. Divergências entre as classificações dos dois codificadores foram analisadas por um terceiro codificador. No entanto, para evitar duplicações na análise, no final foi escolhida apenas a categoria central do trabalho, mesmo que o trabalho tangenciasse outros temas.
Em seguida, para avaliar o perfil metodológico das publicações, foi realizada a leitura dos artigos, em especial dos procedimentos metodológicos, novamente por dois codificadores independentes, previamente treinados. As categorias de análise e os respectivos critérios, utilizados na avaliação dos artigos, foram:

- Tipo de publicação - se era um artigo que apresentava uma discussão puramente conceitual ou se apresentava alguma pesquisa de campo de natureza empírica;

- Abordagem da pesquisa - se a abordagem utilizada era qualitativa, quantitativa ou mista;

- Estratégia de pesquisa - se a pesquisa retratava um estudo de caso, centrado em um contexto organizacional específico, se era um levantamento ou survey, ou se era um experimento laboratorial;

- Procedimentos de coleta de dados - se utilizavam entrevistas, observação, análise documental, questionários ou escalas, ou outros métodos e técnicas de coleta como grupos de foco, testes e provas;

- Procedimentos de análise de dados - se utiliza-

\section{Quadro 1 - Categorias conceituais}

Afeto no trabalho

Aprendizagem organizacional

Atitudes e comportamentos no trabalho

Cognição e percepção no trabalho

Competências

Comunicação

Conflitos e negociação

Cultura e clima organizacional

Desvios de comportamento

Diversidade

Estresse e qualidade de vida no trabalho

Grupos e equipes de trabalho

Liderança

Motivação no trabalho

Mudança organizacional

Relação indivíduo-organização

Tomada de decisão

Valores e personalidade
Satisfação, emoções, prazer, sentimentos e humor

Aprendizagem organizacional e gestão do conhecimento nas organizações

Comportamentos de cidadania e pró-sociais, desempenho, cooperação, participação e rotatividade

Percepções de justiça e equidade, de confiança, de suporte e de política organizacional

Competências gerenciais e individuais e sua gestão

Comunicação interna nas organizações

Gestão de conflitos e resolução de problemas

Crenças, valores, ritos e símbolos organizacionais

Assédio moral e sexual, chantagem, ameaças e corrupção

Gestão de diversidade, gênero, deficiência, orientação sexual e discriminação

Estresse ocupacional, bem-estar físico e psicológico, burnout e segurança no trabalho

Dinâmica e processos de grupos e equipes de trabalho

Teorias de liderança, características do líder e relação superior-subordinado

Práticas motivacionais no ambiente de trabalho

Resistência, reação e adaptação dos indivíduos à mudança

Comprometimento organizacional, contrato psicológico, socialização e identidade

Processo decisório, vieses e racionalidade limitada

Valores individuais e do trabalho e características de personalidade 
vam estatística descritiva, modelagem com teste de relações entre variáveis, análise de conteúdo, análise de discurso ou outras análises interpretativas do tipo etnográfico.

\section{RESULTADOS}

Considerando os artigos publicados em periódicos no período em questão, de um total de 1.895 artigos, 185 foram considerados pertencentes ao campo de CO, o que representa aproximadamente $10 \%$ das publicações. Entre as revistas que mais publicaram artigos de CO, estão a $R A E$, com 52 e a $R A C$, com 43 artigos. Por sua vez, a $R A M$ publicou 37 artigos, e a $R A U S P$ e a $R A P$ publicaram 27 e 26 artigos, respectivamente. A RAM, apesar de só ter publicado artigos de CO a partir de 2004, foi a que apresentou a maior proporção de artigos de $\mathrm{CO}$ em relação ao total publicado no período (13,96\%). Por outro lado, a RAP apresentou o menor percentual de proporção $(5,58 \%)$. Dos anais do EnANPAD, foram selecionados 723 artigos, representando, em média, os mesmos $10 \%$ do total de trabalhos publicados ao longo dos 10 anos (média de 750 trabalhos por edição do evento no período). A Tabela 1 resume o total de artigos publicados por periódico no período analisado, o número de artigos em $\mathrm{CO}$ e a respectiva representatividade.

De modo geral, pode-se concluir que o campo de CO representou cerca de $10 \%$ do total da produção nacional em Administração no Brasil; no entanto, verifica-se um crescimento expressivo do campo ao longo da década, como mostra o Gráfico 1. De 2001 até 2010, a publicação de trabalhos de CO duplicou, o que, em parte, é explicado pelo maior número de trabalhos publicados nos referidos veículos, mas também pelo crescimento do campo.

Em relação aos temas mais estudados pela academia brasileira de Administração, como destaca a Tabela 2, a temática mais pesquisada é cultura organizacional, com 13\% do total de publicação no campo. A maioria dos artigos dessa categoria possui natureza qualitativa e descritiva, relatando estudos de caso. A categoria relação indivíduo-organização (com predomínio de temas como comprometimento organizacional, identidade e contratos psicológicos) foi a segunda mais estudada, seguida pela categoria aprendizagem organizacional. Ambas representam

\section{Tabela 1 - Distribuição dos artigos de CO entre os meios de publicação}

\begin{tabular}{|l|c|c|c|}
\hline Publicação & $\begin{array}{c}\text { Artigos } \\
\text { em CO }\end{array}$ & $\begin{array}{c}\text { Total de } \\
\text { artigos }\end{array}$ & $\begin{array}{c}\text { \% de artigos } \\
\text { em CO }\end{array}$ \\
\hline RAM & 37 & 265 & $13,96 \%$ \\
\hline RAE & 52 & 462 & $11,26 \%$ \\
\hline RAC & 43 & 400 & $10,75 \%$ \\
\hline RAUSP & 27 & 302 & $8,94 \%$ \\
\hline RAP & 26 & 466 & $5,58 \%$ \\
\hline EnANPAD & 723 & 7.500 & $9,64 \%$ \\
\hline Total geral & 908 & 9.395 & $9,66 \%$ \\
\hline
\end{tabular}

\section{Gráfico 1 - Evolução da produção em CO de 2001 a 2010}

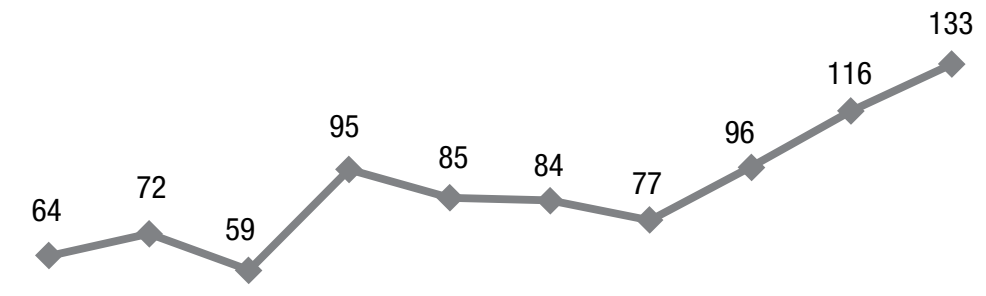

$\begin{array}{llllllllll}2001 & 2002 & 2003 & 2004 & 2005 & 2006 & 2007 & 2008 & 2009 & 2010\end{array}$


$12 \%$ e $11 \%$ da distribuição total, respectivamente, mas diferem quanto ao tipo e estratégia de pesquisa. Enquanto as pesquisas em aprendizagem organizacional seguem o padrão das pesquisas em cultura - qualitativa e baseada em estudos de caso - a categoria relação indivíduo-organização engloba a maioria de artigos com relato de levantamentos de natureza quantitativa. Juntas, as três categorias representam mais de $36 \%$ de toda a produção científica de CO em veículos de Administração no período analisado. Destaque, ainda, para o tema diversidade (predominando artigos sobre discriminação e relações de gênero), com 10\% do total de artigos publicados no período.

\section{Tabela 2 - Distribuição dos artigos de acordo com as categorias de análise}

\begin{tabular}{|l|c|c|c|c|}
\hline \multicolumn{1}{|c|}{ Categorias } & Total & EnANPAD & Periódicos & $\%$ do total \\
\hline Cultura organizacional & 115 & 95 & 20 & $13 \%$ \\
\hline Relação indivíduo-organização & 103 & 78 & 25 & $12 \%$ \\
\hline Aprendizagem organizacional & 94 & 70 & 24 & $11 \%$ \\
\hline Diversidade & 88 & 74 & 14 & $10 \%$ \\
\hline Cognição e percepção no trabalho & 67 & 49 & 18 & $8 \%$ \\
\hline Estresse e qualidade de vida & 57 & 52 & 5 & $6 \%$ \\
\hline Liderança & 57 & 50 & 7 & $6 \%$ \\
\hline Valores e personalidade & 51 & 33 & 18 & $6 \%$ \\
\hline Competências & 49 & 34 & 15 & $6 \%$ \\
\hline Afetos no trabalho & 46 & 37 & 9 & $5 \%$ \\
\hline Mudanças organizacionais & 40 & 35 & 5 & $5 \%$ \\
\hline Atitudes e comportamentos no trabalho & 31 & 23 & 8 & $4 \%$ \\
\hline Motivação & 21 & 16 & 5 & $2 \%$ \\
\hline Comunicação & 19 & 16 & 3 & $2 \%$ \\
\hline Tomada de decisão & 17 & 15 & 2 & $2 \%$ \\
\hline Grupos e equipes de trabalho & 9 & 8 & 1 & $1 \%$ \\
\hline Conflitos e negociação & 9 & 6 & 3 & $1 \%$ \\
\hline Desvios de comportamento & 8 & 5 & 3 & $1 \%$ \\
\hline
\end{tabular}

\section{Gráfico 2 - Evolução das categorias diversidade, relação indivíduo-organização,} valores e personalidade e mudanças organizacionais

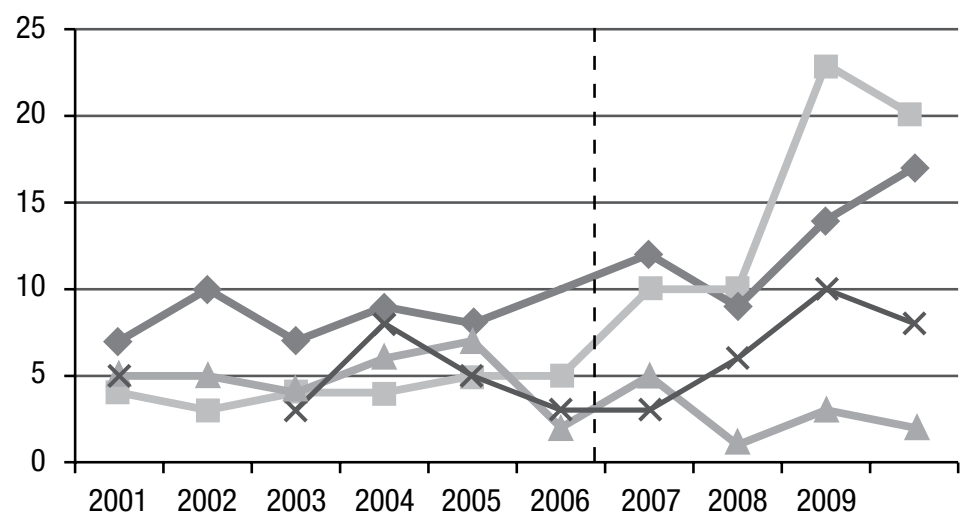

Relação indivíduo-organização

- Diversidade

- Mudanças organizacionais

$* \quad$ Valores e personalidade 
Uma análise temporal evidenciou, ainda, evolução das categorias durante o período analisado. A partir de 2007, o número de artigos publicados com diversidade como tema principal triplicou, mantendo-se nesse nível até 2010 (média de 4,2 artigos de 2001 a 2006; média de 15,8 artigos de 2007 a 2010). Tal crescimento pode ser atribuído a um aumento de estudos sobre gênero, deficiências (inclusão de deficientes) e orientação sexual (homossexualidade e discriminação). Também o número de artigos sobre valores e personalidade teve aumento significativo nos últimos três anos (média de 4,5 artigos de 2001 a 2007; média de 8 artigos de 2008 a 2010). A temática relação indivíduo-organização também apresentou uma tendência ascendente. Nos últimos dois anos, a média de artigos passou de 9 para 16. Ao contrário destas, a categoria mudança organizacional parece estar perdendo espaço. O número de artigos publicados nos últimos 3 anos diminuiu significativamente. O Gráfico 2 apresenta a evolução de produção dos respectivos temas, de 2001 a 2010. As demais categorias analisadas no presente estudo, entretanto, mantiveram-se estáveis no decorrer desses últimos 10 anos.
Quanto à natureza dos trabalhos, $87 \%$ dos artigos publicados nos periódicos são relatos de pesquisas empíricas, enquanto apenas 13\% são discussões teóricas. Em relação à abordagem da pesquisa dos estudos empíricos, percebe-se que há predominância clara de abordagens qualitativas (55\%) em relação às abordagens quantitativas (33\%). Os restantes 12\% dos trabalhos publicados utilizam uma abordagem mista.

A Tabela 3 apresenta a distribuição dos artigos por tema em relação à natureza e abordagem utilizadas, evidenciando uma tendência interessante. Nos temas com uma orientação macro, como cultura, aprendizagem ou mudança, a abordagem qualitativa é predominante, enquanto estudos sobre temas com um enfoque micro, como valores e personalidade, relação indivíduo-organização, atitudes e comportamentos no trabalho, privilegiam abordagens quantitativas.

Considerando ainda os trabalhos empíricos, foram também investigadas as estratégias de pesquisa utilizadas. Os artigos foram classificados como levantamento ou survey, estudo de caso e experimento. Os resultados mostram que, no geral, existe um equilíbrio entre o número de pesquisas que se baseiam em estudos de

\section{Tabela 3 - Distribuição dos artigos por tema, natureza e abordagem de pesquisa}

\begin{tabular}{|c|c|c|c|c|}
\hline \multirow{2}{*}{ Tema } & \multirow{2}{*}{ Teórico } & \multicolumn{3}{|c|}{ Empírico } \\
\hline & & Qualitativo & Quantitativo & Mista \\
\hline Cultura organizacional & 16 & 67 & 27 & 5 \\
\hline Relação indivíduo-organização & 8 & 40 & 44 & 11 \\
\hline Aprendizagem organizacional & 17 & 55 & 15 & 7 \\
\hline Diversidade & 6 & 55 & 14 & 13 \\
\hline Cognição e percepção no trabalho & 6 & 29 & 28 & 4 \\
\hline Estresse e qualidade de vida & 2 & 15 & 24 & 16 \\
\hline Liderança & 10 & 29 & 11 & 7 \\
\hline Valores e personalidade & 6 & 19 & 22 & 4 \\
\hline Competências & 5 & 25 & 10 & 9 \\
\hline Afetos no trabalho & 4 & 20 & 16 & 6 \\
\hline Mudanças organizacionais & 4 & 28 & 3 & 5 \\
\hline Atitudes e comportamentos no trabalho & 4 & 9 & 14 & 4 \\
\hline Motivação & 7 & 4 & 7 & 3 \\
\hline Comunicação & 6 & 11 & 2 & \\
\hline Tomada de decisão & 3 & 6 & 7 & 1 \\
\hline Grupos e equipes de trabalho & 5 & & 3 & 1 \\
\hline Conflitos e negociação & 2 & 3 & 3 & 1 \\
\hline Desvios de comportamento & 1 & 6 & 1 & \\
\hline
\end{tabular}


caso (51\%) e as que utilizam levantamentos do tipo survey (47\%). Por outro lado, as pesquisas que utilizam experimentos (1\%) parecem não possuir qualquer expressividade na pesquisa no Brasil.

Analisando essa distribuição de estratégias de pesquisa ao longo dos 10 anos, nota-se uma tendência decrescente no volume de artigos que conduzem estudos de caso e uma tendência crescente de pesquisas que utilizam surveys como estratégia. Em 2001, $71 \%$ dos trabalhos utilizaram estudos de caso, contra apenas $42 \%$ em 2010, enquanto os levantamentos do tipo survey aumentaram de 29\%, em 2001, para 58\%, em 2010. O Gráfico 3 apresenta a distribuição dos estudos segundo as estratégias de pesquisa utilizadas.

Com relação aos métodos de coleta e análise de dados, as Tabelas 4 e 5 apresentam as técnicas utilizadas por tema. As técnicas de coleta mais utilizadas foram entrevistas e questionários (36\% e 29\%, respectivamente), seguidas de análise documental (17\%) e observação (16\%). Como seria de esperar, temáticas que utilizam abordagens mais qualitativas (como cultura, diversidade e aprendizagem) recorrem a técnicas como entrevistas, análise documental e observação, enquanto pesquisas sobre temas que privilegiam um enfoque mais quantitativo (atitudes e comportamentos no trabalho e valores e personalidade) utilizam predominantemente questionários como técnica de coleta de dados. Dos artigos analisados, 62,9\% utilizam apenas uma técnica de coleta de dados, 28,3\% combinam duas técnicas e apenas 8,8\% combinam três ou mais técnicas. As combinações observação e entrevistas (10,7\%), questionário e entrevistas $(6,9 \%)$ e observação, análise documental e entrevistas (6,2\%) foram as mais significativas, representando técnicas predominantemente aplicadas a estudos de caso.

Já em relação aos procedimentos análise dos dados, como a Tabela 5 mostra, a análise de conteúdo é a técnica mais utilizada, com $49 \%$ de frequência. Em seguida, mas representando apenas 27\% dos trabalhos publicados, vem a modelagem estatística (teste de hipóteses, modelos de regressão ou equações estruturais). Quanto às outras técnicas de análise utilizadas, $16 \%$ dos trabalhos adotam estatísticas descritivas (média, frequência e desvio-padrão) e apenas 6\% utilizam análise de discurso. Cruzando os procedimentos de análise e os temas de $\mathrm{CO}$, os resultados permitem concluir que, de modo geral, a análise de conteúdo é a técnica mais utilizada, independentemente da categoria temática. No entanto, temas com enfoque micro tendem a utilizar mais procedimentos estatísticos na análise dos dados. Em relação à combinação de diferentes tipos de análise, $85,9 \%$ utilizam somente uma técnica, 13,8\% combinam duas técnicas e somente $0,4 \%$ fazem uso de três ou mais técnicas de análise.

Quando se estudam os procedimentos de análise de dados ao longo do período, duas tendências merecem ser destacadas. Como mostra o Gráfico 4, nota-se um crescimento expressivo da utilização de técnicas de modelagem a partir de 2006 (média de 15 artigos de 2001 a 2005; média de 27,5 artigos de 2006 a 2010), parecendo evidenciar uma preocupação com a robustez dos métodos de análise. Por outro lado, os resultados também evidenciam uma tendência

\section{Gráfico 3 - Evolução de utilização de estudos de caso e surveys em pesquisas empíricas}

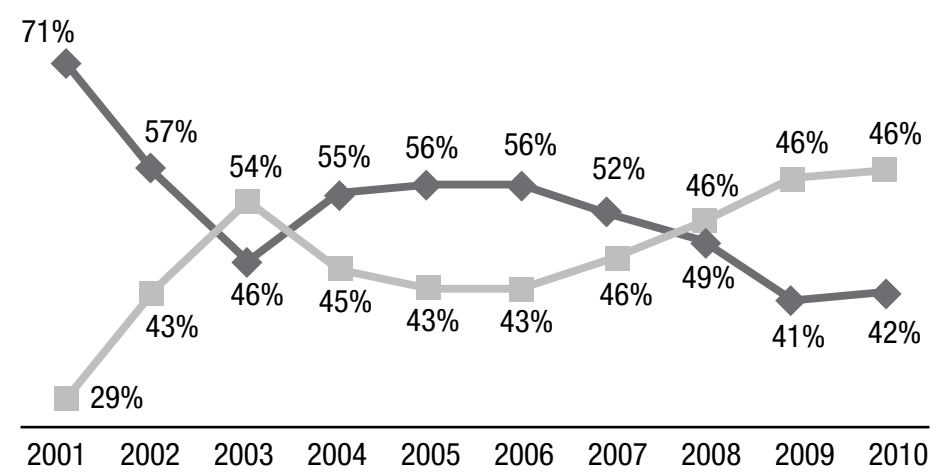


crescente de utilização de triangulação metodológica no decorrer dos 10 anos analisados. Por exemplo, a utilização conjunta de técnicas de estatística descritiva e análise de conteúdo cresceu significativamente a partir de 2008 (média de 4,8 artigos de 2001 a 2007; média de 10,3 artigos de 2008 a 2010).

\section{DISCUSSÃO}

Os resultados apresentados nos permitem identificar algumas tendências e características da produção científica no campo de CO no Brasil. A evolução dos trabalhos em CO mostra que o número de artigos publicados em periódicos possui grande variabilidade: a $R A E$, por exemplo, publicou 13 artigos do campo de CO em 2004, mas apenas 2 no ano seguinte; a $R A C$ não publicou artigos de CO em 2008, mas, em 2009, publicou 4 e, em 2010, 9; da mesma forma, a $R A M$, em 2008, publicou 2 artigos, e nos anos seguintes, 11 e 14, respectivamente. Devido a essa variabilidade, torna-se essencial que sejam analisados os anais do EnANPAD, cuja concentração de artigos chega a ser quatro vezes maior; no entanto, o volume de trabalhos publicados evoluiu linearmente. Apesar da diferença do número total de artigos entre os periódicos e o EnANPAD, o percentual médio de artigos de CO na década é semelhante $(9,7 \%$ e $9,6 \%)$, apontando que a representatividade do campo, independentemente de critérios de aceitação de trabalhos, se situa próximo aos 10\%. Mesmo reconhecendo que é um campo em crescimento no Brasil, essa representatividade nos periódicos de Administração é ainda muito baixa quando comparada com os principais periódicos internacionais da área de Administração. Por exemplo, considerando a mesma década de análise, as publicações em CO representaram mais de $40 \%$ do total de artigos publicados no Academy of Management Journal (MORRISON, 2010).

Os resultados da análise das categorias de CO sugerem uma grande diversidade temática da pro-

\section{Tabela 4 - Quantidade e percentual de utilização de técnicas de coleta de dados dos estudos empíricos por tema}

\begin{tabular}{|c|c|c|c|c|c|}
\hline Temas em CO & $\begin{array}{c}\text { Análise } \\
\text { documental }\end{array}$ & Entrevistas & Observação & Questionários & Outros \\
\hline Cultura organizacional & 37 & 43 & 39 & 33 & 1 \\
\hline Relação indivíduo-organização & 15 & 45 & 16 & 55 & 0 \\
\hline Aprendizagem organizacional & 29 & 50 & 24 & 25 & 2 \\
\hline Diversidade & 18 & 52 & 16 & 21 & 1 \\
\hline Cognição e percepção no trabalho & 8 & 29 & 14 & 32 & 1 \\
\hline Estresse e qualidade de vida & 9 & 25 & 10 & 38 & 1 \\
\hline Liderança & 9 & 30 & 13 & 17 & 1 \\
\hline Valores e personalidade & 6 & 20 & 11 & 27 & 1 \\
\hline Competências & 11 & 27 & 6 & 13 & 1 \\
\hline Afetos no trabalho & 6 & 25 & 7 & 21 & 2 \\
\hline Mudanças organizacionais & 13 & 27 & 8 & 8 & 1 \\
\hline Atitudes e comportamentos no trabalho & 8 & 10 & 6 & 17 & 0 \\
\hline Motivação & 3 & 8 & 0 & 9 & 0 \\
\hline Comunicação & 10 & 4 & 4 & 2 & 0 \\
\hline Tomada de decisão & 2 & 6 & 4 & 4 & 3 \\
\hline Grupos e equipes de trabalho & 1 & 1 & 0 & 3 & 0 \\
\hline Conflitos e negociação & 0 & 2 & 0 & 4 & 1 \\
\hline Desvios de comportamento & 2 & 4 & 1 & 1 & 1 \\
\hline Total & 187 & 408 & 179 & 330 & 17 \\
\hline$\%$ do total & $17 \%$ & $36 \%$ & $16 \%$ & $29 \%$ & $2 \%$ \\
\hline
\end{tabular}


dução científica no campo, embora com alguma predominância de estudos com uma orientação mais macro-organizacional. Importante destacar que a quantidade de trabalhos no nível micro também é bastante expressiva, mas, por estudar fenômenos individuais - bem como os processos por meio dos quais os indivíduos influenciam e são influenciados pelos sistemas organizacionais - é possível que alguns desses trabalhos sejam encaminhados para revistas de Psicologia, reduzindo, assim, o número de artigos publicados sob essa perspectiva na área de Administração.

Tabela 5 - Quantidade e percentual de utilização de técnicas de análise de dados dos estudos empíricos, por tema

\begin{tabular}{|l|c|c|c|c|c|}
\hline \multicolumn{1}{|c|}{ Temas em CO } & $\begin{array}{c}\text { Análise de } \\
\text { conteúdo }\end{array}$ & $\begin{array}{c}\text { Análise de } \\
\text { discurso }\end{array}$ & $\begin{array}{c}\text { Estatística } \\
\text { descritiva }\end{array}$ & Modelagem & Outros \\
\hline Cultura organizacional & 56 & 8 & 13 & 23 & 4 \\
\hline Relação indivíduo-organização & 43 & 5 & 16 & 42 & 0 \\
\hline Aprendizagem organizacional & 52 & 3 & 6 & 20 & 1 \\
\hline Diversidade & 46 & 19 & 19 & 10 & 2 \\
\hline Cognição e percepção no trabalho & 28 & 4 & 4 & 29 & 0 \\
\hline Estresse e qualidade de vida & 27 & 0 & 21 & 21 & 0 \\
\hline Liderança & 34 & 1 & 10 & 8 & 0 \\
\hline Valores e personalidade & 20 & 1 & 10 & 18 & 0 \\
\hline Competências & 31 & 0 & 10 & 10 & 0 \\
\hline Afetos no trabalho & 21 & 3 & 5 & 18 & 0 \\
\hline Mudanças organizacionais & 30 & 1 & 5 & 4 & 0 \\
\hline Atitudes e comportamentos no trabalho & 8 & 1 & 6 & 15 & 0 \\
\hline Motivação & 6 & 1 & 4 & 5 & 0 \\
\hline Comunicação & 9 & 2 & 2 & 1 & 0 \\
\hline Tomada de decisão & 6 & 1 & 3 & 4 & 0 \\
\hline Grupos e equipes de trabalho & 0 & 0 & 1 & 3 & 0 \\
\hline Conflitos e negociação & 3 & 1 & 3 & 1 & 0 \\
\hline Desvios de comportamento & 3 & 3 & 0 & 1 & 0 \\
\hline Total & 423 & 54 & 138 & 233 & 7 \\
\hline \% do total & $49 \%$ & $6 \%$ & $16 \%$ & $27 \%$ & $1 \%$ \\
\hline
\end{tabular}

\section{Gráfico 4 - Evolução de utilização de modelagem e estatística descritiva/análise de conteúdo}

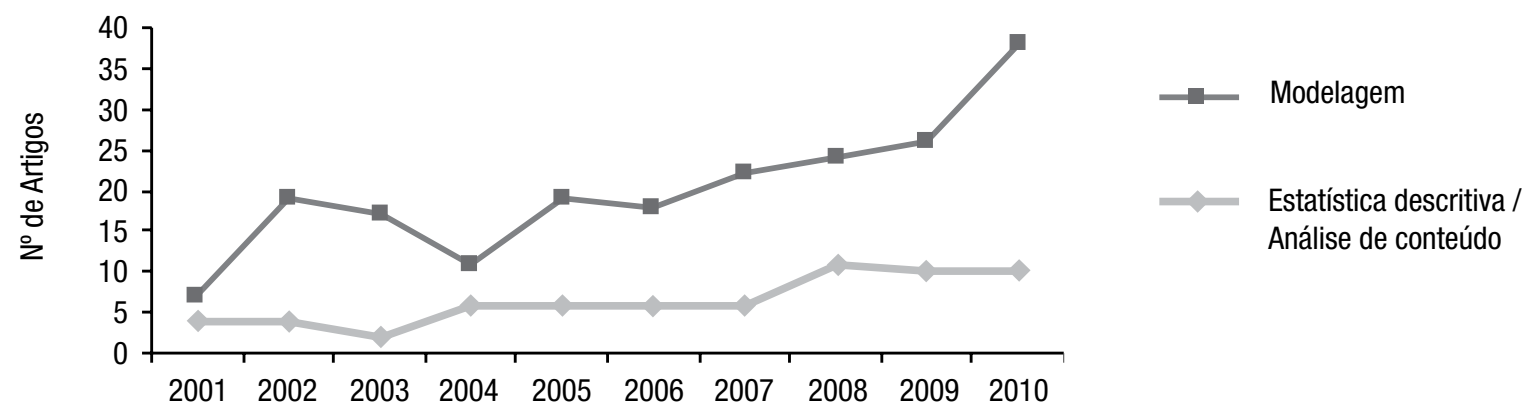


A categoria temática com maior volume de trabalhos foi cultura organizacional. Os trabalhos publicados sobre esse tema tendem a seguir uma abordagem de pesquisa baseada em estudos de caso, de natureza descritiva, que exploram a construção, desconstrução e mudanças na identidade organizacional. Com bastante representatividade, dentro do tema cultura, a temática de valores organizacionais foi explorada na relação com valores pessoais, afetos, comprometimento e saúde e bem-estar.

A segunda categoria mais pesquisada foi a relação indivíduo-organização, com predomínio de trabalhos sobre comprometimento, identidade e contrato psicológico. De maneira geral, essas temáticas já haviam sido identificadas como expoentes na literatura de CO (BORGES-ANDRADE e PAGOTTO, 2010; TONELLI e outros, 2003), indicando certa estabilidade de núcleos de pesquisa no contexto de pós-graduação em Administração no País. As pesquisas nessa temática tendem a apresentar um equilíbrio entre as estratégias qualitativa e quantitativa e não revelam predomínio de nenhum método específico de coleta ou tratamento de dados.

Outra categoria bastante representativa de estudos em CO, a aprendizagem organizacional, também concentrou os seus trabalhos em estudos de caso, focando em gestão do conhecimento, inovação e desempenho organizacional. Pode-se constatar que esse tema apresenta uma tendência constante em termos de volume de publicações, em virtude da sua crescente importância para as organizações e instituições de ensino no Brasil (MACEDO, BOTELHO, DUARTE, 2010).

A análise temporal evidenciou, ainda, a evolução das categorias durante o período analisado, apontando a diversidade como tema em ascendência. Tal crescimento deveu-se a estudos sobre gênero, deficiências e orientação sexual, como verificado em outros trabalhos (TONETTO e outros, 2008). Tudo indica que se trata de núcleos de pesquisadores emergentes que estão incluindo esses temas na pauta de CO.

O número de artigos com valores e personalidade como categoria principal também teve aumento significativo nos últimos 3 anos, com a temática concentrada em valores e crenças pessoais e comportamento ético.

Ao contrário destas, os trabalhos sobre mudança organizacional diminuíram significativamente durante o mesmo período, e demais categorias consideradas no presente estudo mantiveram-se relativamente estáveis no decorrer desses últimos 10 anos.

Finalmente, é importante realçar que as categorias mais investigadas no Brasil diferem substancialmente daquelas publicadas internacionalmente, cujo foco está em grupos e equipes, seguidos por liderança e atitudes e comportamentos no trabalho (MORRISON, 2010). Os resultados sugerem que a produção acadêmica em $\mathrm{CO}$ no Brasil não enfatiza uma perspectiva meso dos fenômenos organizacionais, focando os estudos no nível organizacional ou individual. De fato, o nível meso demanda maior sofisticação teórica e metodológica, que talvez ainda seja distante da área de Administração. Essa ausência de estudos que buscam essa integração entre o nível macro e o nível micro pode evidenciar, ainda, um problema no campo de CO que resulta em uma separação e menor diálogo entre as duas perspectivas dominantes.

Talvez essa diferença possa estar vinculada às diferentes influências da Sociologia e Psicologia nos estudos em $\mathrm{CO}$ no Brasil e no exterior, o que pode esclarecer um pouco a diferença de predominância das temáticas no campo. Tradicionalmente, a análise das organizações no Brasil tem uma raiz sociológica (MOTTA, 2000), ao contrário da literatura internacional de CO, que tem uma influência da Sociologia e a Psicologia. Essa orientação sociológica, reforçada pela predominância de um enfoque macro de pesquisas, reflete-se no perfil metodológico dos trabalhos publicados. A maioria dos trabalhos é qualitativa (54\%), focando essencialmente em estudos de caso (64\%) de natureza descritiva, o que é consistente com resultados de levantamentos de outros campos de Administração no Brasil, que apontam a falta de rigor metodológico como um problema recorrente de produção acadêmica do campo de Administração (BERTERO e KEINERT, 1994; CALDAS e TINOCO, 2004; MACHADO-DA-SILVA, CUNHA, AMBONI, 1990).

Por outro lado, verifica-se o aumento de uma abordagem mista (qualitativa e quantitativa) nos últimos anos, predominantemente com uma metodologia de coleta baseada em questionários estruturados e entrevistas, apontando para uma tendência de consolidação metodológica, talvez decorrente da própria explosão quantitativa do campo acadêmico de Administração, como um todo, e seguindo tendências observadas em outras áreas. O crescimento de estudos que utilizam triangulação de dados ou múltiplas técnicas metodológicas nos leva a crer que os acadêmicos têm procurado investigar e comparar os dados obtidos com maior rigor científico e profundidade. No entanto, apesar do crescimento na utilização de abordagem qualitativa e quantitativa, a predominância ainda é de estudos qualitativos, que se manteve estável durante os 10 anos 
analisados. Tal distribuição difere substancialmente dos estudos internacionais, em que estudos de natureza qualitativa, de natureza monometodológica, são marginais para a maioria dos periódicos que publicam estudos em CO (ROUSSEAU, 2007).

Também vale observar que prevalecem procedimentos de tratamento e análise de dados mais tradicionais, como análise de conteúdo, em vez de técnicas mais sofisticadas, como a análise de discurso ou modelagem estatística. Por outro lado, a utilização de experimentos como estratégia de pesquisa mostrou-se praticamente nula. Mais uma vez, esse resultado mostra uma clivagem grande com a produção científica internacional, em que os estudos experimentais tem maior representatividade no campo de $\mathrm{CO}$ - cerca de $15 \%$ dos artigos de CO no Academy of Management Journal (AMJ) (MORRISON, 2010). Também os estudos com abordagem etnográfica foram inexpressivos. Em 10 anos, somente 7 artigos basearam-se em análises etnográficas, confirmando resultados de estudos anteriores (DAVEL e ALCADIPANI, 2003; TONELLI e outros, 2003; BERTERO, CALDAS, WOOD, 1999).

Quanto aos métodos de coleta dos dados, as entrevistas (36\%) são a técnica mais comum no campo. Como método qualitativo de coleta, as entrevistas exigem rigor para conferir validade aos resultados. No entanto, observam-se várias fragilidades quanto à utilização dessa técnica: muitos estudos não discutem o perfil dos respondentes, não descrevem a condução das entrevistas, os cuidados e critérios que foram utilizados, como se deu a construção do roteiro, entre outros passos metodológicos. Uma especificidade do rigor da abordagem qualitativa refere-se à problemática da validade e da verificação da questão da cientificidade. Poucos foram os estudos que utilizaram métodos de comparação ou triangulação, garantindo validade metodológica. As observações, embora menos utilizadas (16\%), foram, em maioria, do tipo não participante, sendo, de um modo geral, bem explicadas quanto à sua aplicação. Já em relação à análise documental (17\%), raros foram os estudos que descreveram os passos de escolha e estratégia de investigação utilizadas. Por último, em relação à utilização de questionários (29\%), poucos foram os estudos com procedimentos detalhados sobre a aplicação do instrumento. Tais dados expõem o baixo nível do rigor adotado e, logo, algumas fragilidades metodológicas nos trabalhos publicados.

Quanto aos procedimentos de análise de dados, muitos trabalhos revelaram ambiguidades em relação à análise de conteúdo e análise de discurso. Embora 6\% dos estudos tenham afirmado utilizar análise de discurso, alguns artigos descreveram o trabalho analítico como visando as significações de indicadores decodificados, e não como uma leitura com enfoque na posição discursiva do sujeito que produz sentidos. Ou seja, aparentemente, seguem a linha da análise de conteúdo. Por outro lado, poucos estudos utilizaram softwares de análise de dados qualitativos - como Atlas.ti, NVivo, CAQDAS - evidenciando, ainda, uma possível resistência e, ao mesmo tempo, uma embrionária sofisticação metodológica nessas técnicas de análise. Por outro lado, os trabalhos referem-se de maneira genérica à análise de discurso, quando, na prática, trata-se de uma abordagem muito pluralista, em que se destacam correntes como a análise psicológica de discurso, análise crítica de discurso ou análise conversacional, entre tantas outras.

Em relação aos métodos quantitativos, nota-se um aumento da utilização de técnicas de modelagem. Esses estudos priorizam técnicas como a análise fatorial e regressões (simples e múltiplas), revelando alguma sofisticação metodológica em relação a métodos puramente descritivos. Modelos de equações estruturais também têm conquistado espaço significativo no campo de CO nos últimos anos, enquanto outras técnicas (ex.: abordagem multinível) ainda não possuem muita representatividade.

\section{CONCLUSÃO}

O presente estudo buscou analisar a produção científica em CO no campo de Administração no Brasil, com relação aos temas pesquisados, às estratégias de pesquisa e aos procedimentos metodológicos adotados pelos pesquisadores no período de 2001 a 2010. O levantamento realizado e os resultados encontrados contribuem para uma melhor delimitação do campo de CO e caracterização do seu escopo temático e opções metodológicas.

A pesquisa demonstra que o campo de CO ainda representa uma pequena parcela das publicações em estudos organizacionais no Brasil. Entretanto, o campo possui uma temática bastante abrangente, fruto da multidisciplinaridade das suas influências, porém distinta dos estudos internacionais, mesclando estudos com foco predominantemente na organização (nível macro), mas também no indivíduo (nível micro). 
Destaca-se uma menor ênfase a temas de nível grupal (equipes e conflito) e relacional (liderança). A persistência de alguns temas, como aprendizagem ou comprometimento organizacional, parece estar relacionada com a existência de núcleos consolidados de pesquisadores no País, assim como a emergência de temas novos, como valores humanos e diversidade, pode indicar a institucionalização de outros. Pesquisas futuras poderiam explorar as relações entre o conhecimento produzido em $\mathrm{CO}$ e os núcleos institucionais de pesquisadores no Brasil, aprofundando a pesquisa realizada por Guimarães e outros (2009).

A pesquisa mostrou, ainda, que, na década analisada, no Brasil, a produção científica em CO possui uma identidade própria, porém num estágio inicial de evolução: embora tenha apresentado caráter predominantemente qualitativo e com técnicas de análise pouco robustas, apresentou indícios de um aumento crescente de volume, maior variabilidade de estratégias de pesquisa e, ainda, um aumento da sofisticação de técnicas de análise de dados, acompanhando o aumento quantitativo do campo e seguindo tendências observadas nas avaliações de outras áreas de conhecimento.

Vale apontar que os dados analisados na presente pesquisa trazem limitações em relação à coleta e organização dos dados, uma vez que as bases de dados foram os principais periódicos de Administração e os anais do EnANPAD. Sabe-se que parte da produção intelectual em CO está divulgada em revistas de psicologia, entretanto buscou-se, deliberadamente, neste trabalho analisar como a área de Administração aborda o tema de CO. Afinal, trata-se de uma área definida como multidisciplinar, entretanto a multidisciplinaridade pode tornar-se um caminho mais curto para a multissuperficialidade temática e metodológica, que pode significar uma fragilidade do campo.

\section{PROPOSTAS PARA O FUTURO}

A participação de publicações nos periódicos e nos anais dos EnANPADs indica que ainda se encontram em níveis inferiores àqueles que são encontrados internacionalmente. Portanto, seria de todo desejável maior ênfase na área, mormente quando se observam as fragilidades na formação de administradores nas chamadas soft skills.
Ainda conviria observar que, devido ao viés metodológico dos estudos realizados, há necessidade de se ampliarem as pesquisas no que diz respeito à escolha epistemológica e utilização de métodos e técnicas adequadas a ela, o que garantiria maior validade científica e, portanto, maior contribuição para o campo de CO.

Paralelamente, poderia haver uma intensificação de estudos no nível meso e utilização de abordagens em vários níveis, além do aumento de estudos inferenciais, com base em experimentos e testes controlados. Para a consolidação do campo nos próximos anos, as pesquisas deveriam, ainda, adotar perspectivas mais amplas em relação a questões teórico-metodológicas, submetendo teorias a testes empíricos mais robustos. A literatura indica que o COadotou internacionalmente uma metodologia preferencialmente quantitativa, enquanto a produção científica nacional analisada indica preferência por metodologia qualitativa. Seria aconselhável que se buscasse uma produção mais equilibrada, usando ambas as metodologias.

\section{REFERÊNCIAS}

BASTOS, A. V. B; FRANÇA, A; PINHO, A. P. M.; PEREIRA, L. Pesquisa em comportamento organizacional no Brasil: o que foi divulgado nos nossos periódicos científicos? In: Sociedade Interamericana de Psicologia (Ed.), Anais, XXVI Congresso Interamericano de Psicologia. São Paulo, SP: Sociedade Interamericana de Psicologia, 1997.

BERTERO, C. O; CALDAS, M; WOOD, T. Produção científica em Administração de empresas: provocações, insinuações e contribuições para um debate local. Revista de Administração Contemporânea, v. 3, n. 1, p. 147-178, 1999.

BERTERO, C. O; KEINERT, T. M. M. A evolução da análise organizacional no Brasil (1961-93). RAE-Revista de Administração de Empresas,, v. 34, n. 3, p. 81-90, 1994.

BORGES-ANDRADE, J. E; OLIVEIRA-SOUZA, C. M; PILATI, R; NONATO, A. J; SIlvinO, A. M. D; \& GAMA, A. L. G. Pesquisa em comportamento organizacional no Brasil: Que fizeram nossas pós-graduações e que estão fazendo nossos pesquisadores? In Sociedade Interamericana de Psicologia (Ed.), Anais, XXVI Congresso Interamericano de Psicologia, São Paulo, SP: Sociedade Interamericana de Psicologia, 1997. 
BORGES-ANDRADE, J. E; PAGOTTO, C. P. O estado da arte da pesquisa brasileira em psicologia do trabalho e organizacional. Psicologia: teoria e pesquisa [on-line], v. 26, Edição Especial, p. 37-50, 2010.

CALDAS, M; TINOCO, T. Pesquisa em gestão de recursos humanos nos anos 1990: um estudo bibliométrico. $R A E-$ -Revista de Administração de Empresas, v. 44, n. 3, p. 100-114, 2004.

DAVEL, E; ALCADIPANI, R. Estudos críticos em Administração: a produção científica brasileira nos anos 1990. RAE-Revista de Administração de Empresas, v. 43, n. 4, p. 72-85, 2003.

GUIMARÃES, T. A; GOMES, A. O; ODELIUS, C. C; ZANCAN, C; CORRADI, A. A. A rede de programas de pós-graduação em Administração no Brasil: análise de relações acadêmicas e atributos de programas. Revista de Administração Contemporânea, v. 13, p. 564-582, 2009.

MACEDO, M; BOTELHO, L. L. R; DUARTE, M. A. T. Revisão bibliométrica sobre a produção científica em aprendizagem gerencial. GES - Revista Gestão e Sociedade CEPEAD/ UFMG, v. 4, n. 8, p. 619-639, 2010.

MACHADO-DA-SILVA, C. L; CUNHA,V. C; AMBONI, N. Organizações: o estado da arte da produção acadêmica no Brasil. In: Encontro Nacional da Associação Nacional de Programas de Pós-graduação em Administração, 14, 1990. Anais. ANPAD. Florianópolis: ANPAD, 1990.

MITCHELL, T. R. Organizational behavior. Annual Review of Psychology, v. 30, p. 243-281, 1979.

MORRISON, E. From the editors. OB in AMJ: what is hot and what is not? Academy of Management Journal, v. 53, n. 5, p. 932-936, 2010.

MOTTA, F. C. P. Os pressupostos básicos de Schein e a fronteira entre a psicanálise e a cultura organizacional. In: PRESTES MOTTA, F. C; FREITAS, M. E. Vida psíquica e organização. Rio de Janeiro: FGV, 2000.

MOWDAY, R. T; SUTTON, R. I. Organizational behavior: linking individuals and groups to organizational contexts. Annual Review of Psychology, v. 44, p. 195-229, 1993.

NORTHCRAFT, G. B; NEALE M. A. Organizational behavior: a management challenge. Chicago: Dryden Press, 1990.
O'REILLY, C. A. Organizational behavior: where we've been, where we're going. Annual Review of Psychology, 42, p. 427-458, 1991.

PAYNE, R; PUGH, D. S. Organizations as psychological environments. In: WARR, P. B. (Org). Psychology at work. Harmondsworth: Penguin, 1971. p. 374-402.

PUGH, D. S. Modern organization theory: a psychological and sociological study. Psychological Bulletin, v. 66, n. 4 , p. 235-251, 1966.

PUGH, D. S. Organization behavior: an approach from psychology. Human Relations, v. 22, n. 4, p. 345-354, 1969.

ROUSSEAU, D. M. Organizational behavior in the new organizational era. Annual Review of Psychology, v. 48, p. 515-546, 1997.

ROUSSEAU, D. M. Standing out in the fields of organization science. Journal of Organizational Behavior, 28, n. 7, p. 849-857, 2007.

SIQUEIRA, M. M. M. Medidas do comportamento organizacional. Estudos de Psicologia, v. 7, spe, p. 11-18, 2002.

STAW, B. M. Organizational behavior: a review and reformulation of the field's outcome variables. Annual Review of Psychology, v. 35, p. 627-666, 1984.

TONELLI, M. J; CALDAS, M. P; LACOMBE, B. M. B; TINOCO, T. Produção acadêmica em recursos humanos no Brasil: 1991-2000. RAE-Revista de Administração de Empresas, v. 43, n. 1, p. 105-122, 2003.

TONETTO, A. M; AMAZARRAY, M. R; KOLlER, S. H; GOMES, W. B. Psicologia organizacional e do trabalho no Brasil: desenvolvimento científico contemporâneo. Psicologia E Sociedade, v. 20, n. 2, p. 165-173, 2008. 